\title{
MicroRNA-138-5p regulates neural stem cell proliferation and differentiation in vitro by targeting TRIP6 expression
}

\author{
JUAN WANG ${ }^{1,2}$, JIXIA LI $^{3}$, JIAN YANG ${ }^{4}$, LIANGUO ZHANG ${ }^{5}$, \\ SHANE GAO $^{1}$, FEI JIAO ${ }^{2}$, MAOLI YI $^{6}$ and JUN XU ${ }^{1,2}$ \\ ${ }^{1}$ Stem Cell Center, East Hospital, Tongji University School of Medicine, Shanghai 200120; \\ ${ }^{2}$ Department of Biotechnology and Molecular, Binzhou Medical College, Yantai, Shandong 264003; \\ ${ }^{3}$ Clinical Laboratory, Yantaishan Hospital; ${ }^{4}$ Administration Office, Yantai Blood Station, Yantai, Shandong 264000; \\ ${ }^{5}$ Department of Thoracic Surgery, Affiliated Hospital of Binzhou Medical College, Binzhou, Shandong 256603; \\ ${ }^{6}$ Laboratory of Yantai Yuhuangding Hospital, Yantai, Shandong 264003, P.R. China
}

Received September 27, 2016; Accepted July 5, 2017

DOI: $10.3892 / \mathrm{mmr} .2017 .7504$

\begin{abstract}
Research on neural stem cells (NSCs) has recently focused on microRNAs (miRNAs), a class of small non-coding RNAs that have crucial roles in regulating NSC proliferation and differentiation. In the present study, a quantitative-polymerase chain reaction assay revealed that the expression of miRNA (miR)-138-5p was significantly decreased during neural differentiation of NSCs in vitro. Overexpression of miR-138-5p reduced NSC proliferation and increased NSC differentiation. Furthermore, suppression of miR-138-5p via transfection with a miRNA inhibitor enhanced NSC proliferation and attenuated NSC differentiation. Additionally, expression of thyroid hormone receptor interacting protein 6 (TRIP6), a critical regulator of NSCs, was negatively correlated with the miR-138-5p level. A luciferase assay demonstrated that miR-138-5p regulate TRIP6 by directly binding the 3 '-untranslated region of the mRNA. Additionally, upregulation of TRIP6 rescued the NSC proliferation deficiency induced by miR-138-5p and abolished miR-138-5p-promoted NSCs differentiation. By contrast, downregulation of TRIP6 produced the opposite effect on proliferation and differentiation of NSCs transfected with anti-miR-138-5p. Taken together, the data suggest that miR-138-5p regulates NSCs proliferation and differentiation, and may be useful in developing novel treatments for neurological disorders via manipulation of miR-138-5p in NSCs.
\end{abstract}

Correspondence to: Professor Jun Xu, Stem Cell Center, East Hospital, Tongji University School of Medicine, 150 Jimo Road, Shanghai 200120, P.R. China

E-mail:pandelaila@163.com

Professor Maoli Yi, Laboratory of Yantai Yuhuangding Hospital, 20 East Yuhuangding Road, Yantai, Shandong 264003, P.R. China E-mail: giczpk@sina.com

Key words: proliferation, differentiation, miR-138-5p, neural stem cells, thyroid hormone receptor interacting protein 6

\section{Introduction}

Neural stem cells (NSCs) are self-renewing, multipotent and undifferentiated precursors that retain the ability to differentiate into glial and neuronal lineages, which offer potential for use in cell-based therapy strategies for neurological disorders, including Alzheimer's disease, Parkinson's disease, Huntington's disease and nerve damage (1-3). Given the importance of NSCs in the development of the nervous system, it has been proposed that the use of NSCs for the treatment of neurodevelopmental disorders may be a promising approach to rescue impaired neuronal plasticity $(4,5)$. Additionally, neurogenesis is tightly regulated at multiple levels by extrinsic and intrinsic factors and uncovering the molecular mechanisms that underlie neurogenesis is crucial to understand the functions and plasticity of brain development, and to prevent such pathologies. Recent studies achieved substantial progress, which has been made in elucidating the regulatory mechanisms underlying NSC proliferation, differentiation and functional integration in neural circuits (6,7).

It has been previously reported that microRNAs (miRNAs), a subset of small, noncoding RNAs, can directly bind to the 3' untranslated region (3'UTR) of mRNAs to regulate gene expression post-transcriptionally resulting in translational repression or mRNA degradation (8). miRNAs are involved in a considerable variety of biological process, including apoptosis, proliferation, differentiation and survival. Increasing evidence suggests that miRNAs have important roles in neuronal differentiation, maturation, and synaptic function, including neural stem cell proliferation and differentiation (9). As demonstrated in previous studies, aberrant expression of miRNAs is closely associated with brain disease, and ectopic expression of specific miRNAs may modulate NSCs biological function (10). However, the precise regulatory mechanisms of miRNAs in NSCs remain largely unexplored.

The aim of the present study was to determine the role of miRNA (miR)-138-5p in NSCs proliferation and differentiation. The expression of miR-138-5p was examined during neuronal differentiation of NSCs in vitro and evaluated the function and the induction mechanism. 


\section{Materials and methods}

Cell culture. By using the Percoll gradient method described previously, NSCs were stemmed from adult (8-10 week old) male C57BL/6 mouse forebrain, supplied by the Laboratory Animal Center of Tongji University (Shanghai, China) (11). The cells were cultured in DMEM/F12 medium (Invitrogen; Thermo Fisher Scientific, Inc., Waltham, MA, USA) supplemented with $1 \mathrm{mM}$ L-glutamine (Sigma-Aldrich; Merck KGaA, Darmstadt, Germany), $1 \% \mathrm{~N}_{2}$ supplement (Gibco; Thermo Fisher Scientific, Inc.), $20 \mathrm{ng} / \mathrm{ml}$ epidermal growth factor (EGF; PeproTech, Inc., Rocky Hill, NJ, USA), $20 \mathrm{ng} / \mathrm{ml}$ basic fibroblast growth factor (bFGF; PeproTech, Inc.), $50 \mathrm{ng} / \mathrm{ml}$ heparin (Sigma-Aldrich; Merck KGaA) and 1\% penicillin/streptomycin (Invitrogen; Thermo Fisher Scientific, Inc.) in humidified air at $37^{\circ} \mathrm{C}$ and $5 \% \mathrm{CO}_{2}$. For neural differentiation, NSCs were cultured in an environment with $1 \mathrm{mM}$ retinoic acid (Sigma-Aldrich; Merck KGaA) and $0.5 \%$ foetal bovine serum (FBS) for 3 days in $0.5 \% \mathrm{~N}_{2}$ with Euromed-N medium (Invitrogen; Thermo Fisher Scientific, Inc.) to induce neural differentiation. This study was approved by the Ethics Committee of East Hospital, Tongji University School of Medicine (no. EHTJ2016022; Shanghai, China).

Cell transfection. The miR-138-5p (5'-AGCUGGUGUUGU GAAUCAGGCCG-3')/anti-miR-138-5p mimics (5'-CGG CCTGATTCACAACACCAGCT-3') and its control mimics (5'-ATTTAGCCGGTACATCAGGCC-3') were purchased from Shanghai GenePharma Co., Ltd (Shanghai, China). NSCs were transfected with the miRNA mimic $(50 \mathrm{nM})$ using Dharmafect 1 (GE Dharmacon; GE Healthcare Life Sciences, Lafayette, CO, USA) according to the manufacturer's instructions following seeding of $1 \times 10^{5} /$ well in 6 -well plates and cultured to $70 \%$ confluence. Cells were collected for further analyses $48 \mathrm{~h}$ after transfection.

$R N A$ extraction and reverse transcription-quantitative polymerase chain reaction (RT- $q P C R$ ). Total RNAs including mRNAs and small RNAs from NSCs cells were extracted using TRIzol reagent (Invitrogen; Thermo Fisher Scientific, Inc.). Complementary DNA (cDNA) was generated using TaqMan microRNA RT kit (Applied Biosystems; Thermo Fisher Scientific, Inc.) for miRNAs and SuperScript VILO cDNA synthesis kit (Thermo Fisher Scientific, Inc.) for mRNAs. qPCR was performed using SYBR Green PCR kit (Qiagen China Co., Ltd., Shanghai, China) on a 7900 Real-time PCR System (Applied Biosystems; Thermo Fisher Scientific, Inc.). PCR was performed with the following thermocycling conditions: 25 cycles of $10 \mathrm{sec}$ at $98^{\circ} \mathrm{C}, 10 \mathrm{sec}$ at $55^{\circ} \mathrm{C}$ and $20 \mathrm{sec}$ at $72^{\circ} \mathrm{C}$. The primers used were as follows: Nestin, forward 5'-GATCTAAACAGGAAGGAAATCCAGG-3' and reverse 5'-TCTAGTGTCTCATGGCTCTGGTTTT-3'; GFAP, forward 5'-CAACGTTAAGCTAGCCCTGGACAT-3' and reverse 5'-CTCACCATCCCGCATCTCCACAGT-3'; neuronal class III $\beta$-tubulin (Tuj1), forward 5'-CGCCATGTT CAGACGCAAG-3' and reverse 5'-CTCGGACACCAGGTC GTTCA-3'; GAPDH, forward 5'-ATTCCATGGCACCGT CAAGGCTGA-3' and reverse 5'-TTCTCCATGGTGGTG AAGACGCCA-3'; miR-138-5p, forward 5'-AGCTGGTGT TGTGAATCAGGCCG-3' and reverse 5'-TGGTGTCGTGGA
GTCG-3'; U6, forward 5'-CTCGCTTCGGCAGCACA-3' and reverse 5'-AACGCTTCACGAAYYYGCGT-3'. The usage of the $2^{-\triangle \Delta C q}$ method (12) calculated the relative quantification. By using GAPDH/U6 as a control for mRNA and miRNA respectively, a series of data has been standardized.

Immunofluorescence. NSCs were fixed in 4\% paraformaldehyde for $24 \mathrm{~h}$ at room temperature and then permeabilized by using $0.2 \%$ Triton-X for $1 \mathrm{~h}$ at room temperature. Following blocking with 10\% goat serum (Invitrogen; Thermo Fisher Scientific, Inc.) for $1 \mathrm{~h}$ at room temperature, cells were treated with rabbit anti-Ki67 (cat. no. 612254; 1:250; BD Biosciences, Franklin Lakes, NJ, USA) and anti-thyroid hormone receptor interactor 6 (TRIP6; cat. no. 612254; 1:250; BD Bioscience, Franklin Lakes, NJ, USA) antibodies at $4^{\circ} \mathrm{C}$ overnight, followed by incubation with DyLight-549 goat anti-rabbit antibody (cat. no. 012-500-003; 1:500; Jackson ImmunoResearch, West Grove, PA) according to the manufacturer's instructions. Sections were washed with PBS and incubated with $0.5 \mu \mathrm{g} / \mathrm{ml}$ 4,6-Diamidino-2-phenylindole (DAPI; Vector Laboratories, Inc., Burlingame, CA, USA) solution for $30 \mathrm{~min}$ in the dark at room temperature; DAPI was used for nuclear staining in order to maintain an analogous background. Cells were imaged using an inverted fluorescence microscope (Leica DMI3000; Leica Microsystems, Inc.). The labelled nuclei and fluorescence intensity were analysed in an average of 5 high-powered fields (magnification, x200) using ImageJ software (version 1.48; National Institutes of Health, Bethesda, MD, USA).

Western blot assays. Cells were lysed on ice using $1 \mathrm{X}$ SDS lysis buffer [100 mM 2-ME, $50 \mathrm{mM}$ Tris-HCl (pH 6.8), 2\% w/v SDS, $10 \%$ glycerol]. Protein concentration was determined using the bicinchoninic acid method. Protein $(30 \mu \mathrm{g})$ was separated by $10-12 \%$ SDS-PAGE and transferred to nitrocellulose membrane (GE Healthcare Life Sciences). Membranes were probed with anti-GAPDH antibody (cat. no. \#sc-25778; 1:2,000; Santa Cruz Biotechnology, Inc.) and anti-TRIP6 antibody (cat. no. 612254; 1:500; BD Bioscience) overnight at $4^{\circ} \mathrm{C}$ and then incubated with the horseradish peroxidase-conjugated secondary antibodies (cat. no. 1662408; 1:3,000; Bio-Rad Laboratories Inc., Hercules, CA, USA) at $37^{\circ} \mathrm{C}$ for $1 \mathrm{~h}$. The bands were visualized with the ChemiDoc XRS system (Bio-Rad Laboratories, Inc.).

miRNA target analysis. Genes containing the miR-binding sites in the UTR were identified using TargetScan Release 7.1 (www.targetscan.org/vert_71/).

Luciferase reporter assays. The full-length 3'-untranslated region of TRIP6 was synthesized by PCR from human cDNA [obtained from the U87 cell line; American Type Culture Collection (ATCC), Manassas, VA, USA]. The primers were as follows: Forward, 5'-GTCTTCCTAGAA GTACC-3'; reverse, 5'-CGAGGGATTATTATTTC-3'. The PCR product was cloned into the pmirGLO vector luciferase reporter (Promega Corporation, Madison, WI, USA). For point mutation, site-directed mutagenesis of potential target site in the TRIP6 3'UTR was performed using a QuikChange Site-Directed Mutagenesis kit (Promega Corporation; primers: Forward, 5'-GATCTGGGCTGCGACGGCC-3'; 
A

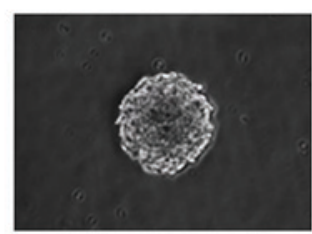

Day 0

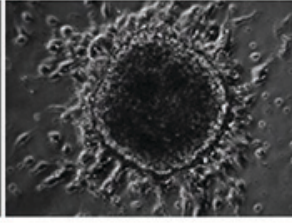

Day 3

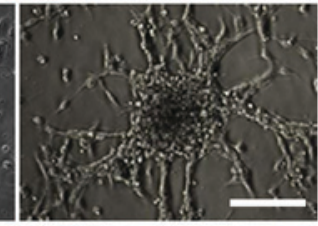

Day 7
B

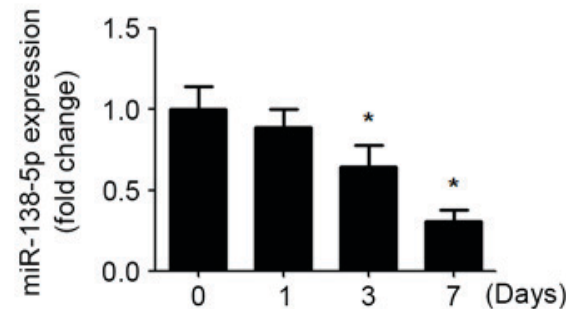

Figure 1. Decreased miR-138-5p level in differentiated neural stem cells. (A) Dissociated neurospheres were observed by inverted microscope during differentiation (scale bar, $50 \mu \mathrm{m}$ ). (B) Expression of miR-138-5p was measured at day $0,1,3,7$ by quantitative polymerase chain reaction analysis. The data are expressed as the mean \pm standard deviation. ${ }^{*} \mathrm{P}<0.05$ vs. day $0, \mathrm{n}=5$. $\mathrm{miR}$, microRNA.

reverse, 5'-GGCCGTCGCAGCCCAGATC-3'). The recombinant plasmids containing wildtype/mutant 3'-UTR of TRIP6 were confirmed by sequencing. The NSCs cells were cultured to $70-80 \%$ confluence in 24 -well plates and co-transfected with a firefly luciferase reporter vector containing the TRIP6 3'UTR or mutant 3'UTR and miR-138-5p/anti-miR-138-5p or control mimics $(50 \mathrm{nM})$ using Lipofectamine ${ }^{\mathrm{TM}} 2000$ reagent (Invitrogen; Thermo Fisher Scientific, Inc., Waltham, MA, USA). The control luciferase gene was also on this empty vector. Luciferase activity was analysed $48 \mathrm{~h}$ after cotransfection using a dual-luciferase reporter system (Promega Corporation).

Plasmid construction. For the TRIP6-overexpressing construct, a human cDNA (U87 cell line; ATCC) sequence of mouse TRIP6 was inserted into the T expressing vector with CMV promoter (Promega Corporation, primer, forward, 5'-ATGTCGGGGCCCACCTGGCT-3'; reverse, 5'-TCAGCA GTCAGTGGTGACGGT-3'). TRIP6-targeting short hairpin RNA (shTRIP6; GCCTGGACGCCGAGATAGA) was synthesized by Shanghai GenePharma Co., Ltd and inserted in pSUPER vector (OligoEngine, Seattle, WA, USA). NSCs were seeded at a density of $70 \%$ confluence. NSCs were transfected with the plasmid $(50 \mathrm{nM})$ using Dharmafect 1 (GE Dharmacon; GE Healthcare Life Sciences) according to the manufacturer's instructions. In addition, NSCs were co-transfected with the miR-138-5p+ctrl vector, miR-138-5p+TRIP6 plasmid, anti-miR-138-5p+sh-ctrl vector or anti-miR-138-5p+sh-TRIP6 plasmid at $37^{\circ} \mathrm{C}$ for $48 \mathrm{~h}$. Cells were collected for further analyses performed $48 \mathrm{~h}$ after transfection.

Statistical analysis. Statistical analyses were processed using SPSS version 11.5 (SPSS Inc., Chicago, IL, USA). The data are presented as the mean \pm standard deviation of at least three independent experiments. Significant differences were analysed using Student's t-test between two groups, and one-way analysis of variance was used for multiple comparisons. A Tukey post hoc test was used to compare the differences between three groups. $\mathrm{P}<0.05$ was considered to indicate a statistically significant difference.

\section{Results}

miR-138-5p is downregulated during NSCs differentiation. In order to determine the involvement of miR-138-5p in regulating NSC differentiation, an in vitro model of NSC differentiation was established. NSCs obtained from mouse forebrain formed neurospheres in the presence of EGF and bFGF. Dissociated neurospheres were re-seeded and differentiated in EGF- and bFGF-free medium. After 7 day of differentiation, the NSCs developed neuronal morphology with long and branched neurites (Fig. 1A). Subsequently, miR-138-5p expression was detected during NSC differentiation. During NSC differentiation, the expression of miR-138-5p was gradually decreased over time (Fig. 1B).

miR-138-5p regulates the proliferation and differentiation of NSCs. To investigate the functional implications of miR-138-5p in NSCs, the effect of miR-138-5p overexpression or silencing on NSCs proliferation and differentiation was determined (Fig. 2A). As detected by the Ki67 immunofluorescence assay, the results demonstrated that overexpression of miR-138-5p reduced NSC proliferation, whereas suppression of miR-138-5p enhanced NSCs proliferation (Fig. 2B). Furthermore, miR-138-5p overexpression significantly promoted expression of the neuronal marker Tuj1 and decreased expression of nestin, a neural stem cell marker, in NSCs induced to differentiate with retinoic acid and FBS (Fig. 2C). By contrast, suppression of miR-378 presented exactly the opposite effect on NSCs differentiation (Fig. 2C).

TRIP6 is a direct target of miR-138-5p in NSCs. Recent studies have demonstrated that TRIP6 regulates neural stem cell maintenance and serves as a new marker for NSCs $(13,14)$. Western blot analysis showed that the expression of TRIP6 was increased the same time as post NSCs differentiation (Fig. 3A). Bioinformatic algorithms (TargetScan) indicated that TRIP6 is a potential target of miR-138-5p. Correlation analysis demonstrated that miR-138-5p expression was inversely correlated with TRIP6 level during NSCs differentiation on days $0,1,3$ and 7 (Fig. 3B). Immunofluorescence indicated that increased miR-138-5p reduced TRIP6 expression, and downregulation of miR-138-5p level elevated TRIP6 expression (Fig. 3C). To verify whether miR-138-5p could directly bind the 3'UTR of TRIP6, we analysed the potential seed sequence for miR-138-5p in the 3'UTR region of TRIP6 mRNA and cloned the wild type and mutant TRIP6 3'UTR fragments into a luciferase reporter gene system (Fig. 3D). The miR-138-5p/anti-miR-138-5p mimic and the redesigned luciferase reporter plasmid were then co-transfected into NSCs cells. Luciferase activity from the wild type vector was increased by knockdown of miR-138-5p and reduced by 


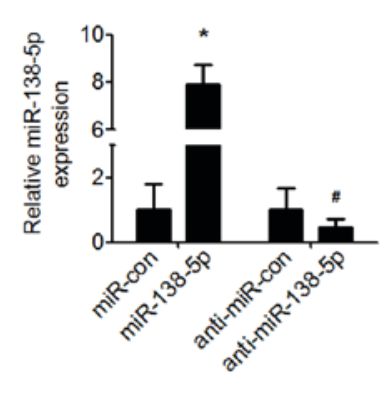

B

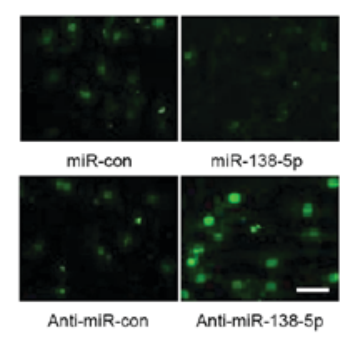

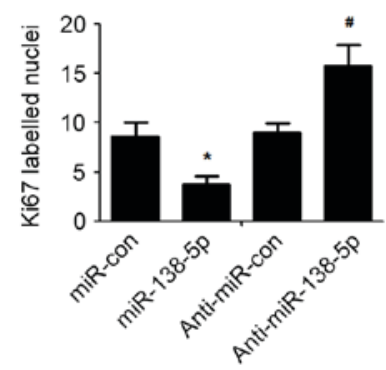

C
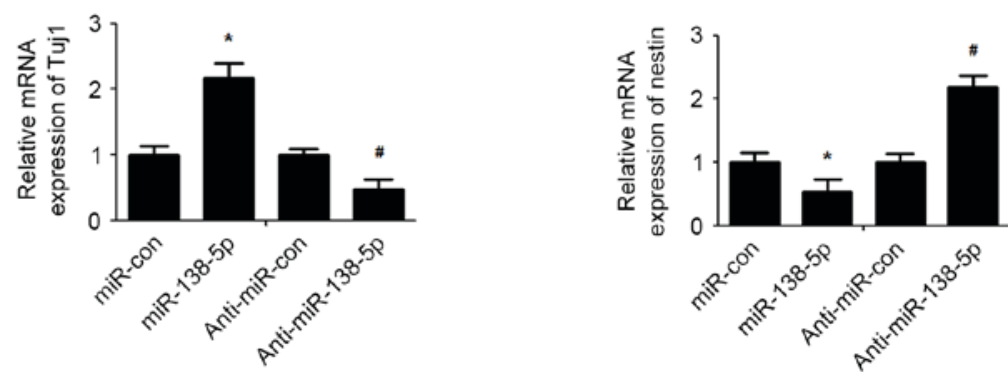

Figure 2. miR-138-5p regulates NSCs proliferation and differentiation. (A) NSCs were transfected with the miR-138-5p/anti-miR-138-5p or control mimic/ inhibitor for 48 h. (B) Proliferation of NSCs was detected by the Ki67 immunofluorescence assay (scale bar, $50 \mu \mathrm{m}$ ). Quantification of the number of Ki67 labelled nuclei was performed by counting five high-power fields. (C) Tuj1 and nestin expression was detected by quantitative polymerase chain reaction analysis in NSCs following treatment with $1 \mathrm{mM}$ retinoic acid and $0.5 \%$ foetal bovine serum for 3 days. The data are expressed as the mean \pm standard deviation. ${ }^{\text {"P }}<0.05$ vs. miR-con group, ${ }^{~} \mathrm{P}<0.05$ vs. anti-miR-con group, $\mathrm{n}=5$. NSCS, neural stem cells; miR, microRNA; miR-con, control miR; Tuj1, class III $\beta$-tubulin.

A
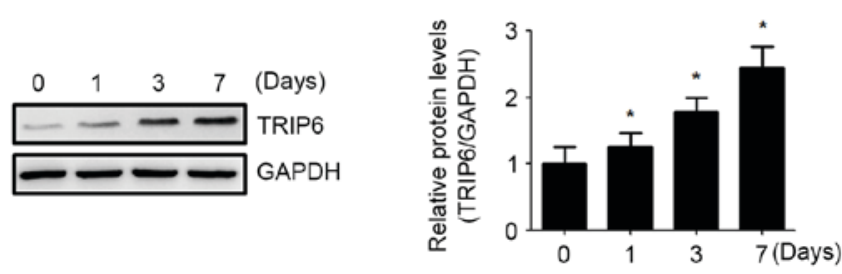

C

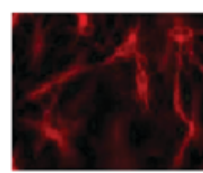

miR-con

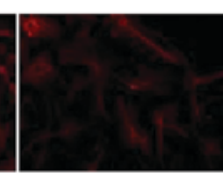

miR-138-5p

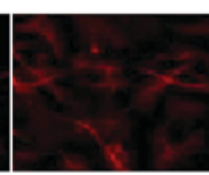

Anti-miR-con

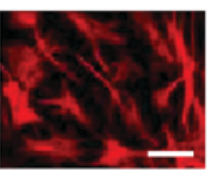

Anti-miR-138-5p
B
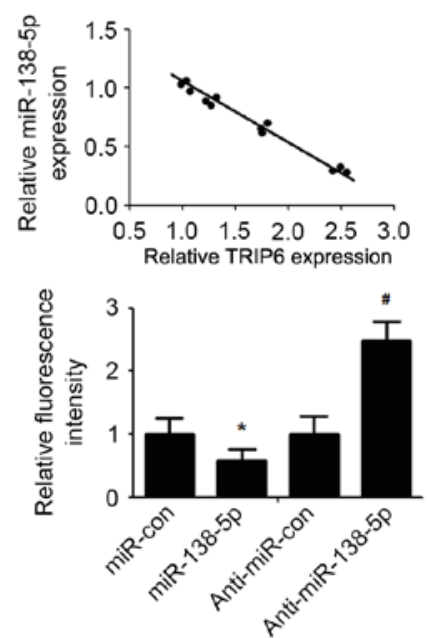

D

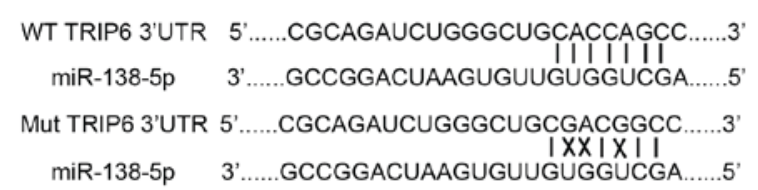

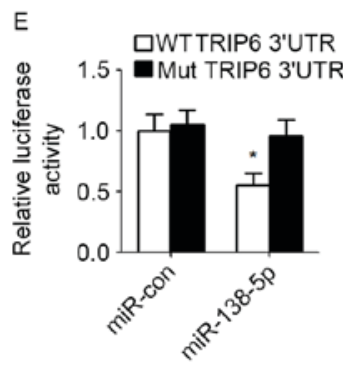

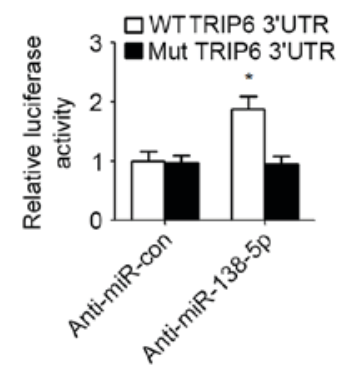

Figure 3. miR-138-5p regulates TRIP6 expression by directly binding its 3'UTR. (A) TRIP6 protein level was detected by western blot in NSCs during differentiation. Quantification of relative protein levels (TRIP6/GAPDH) according to band intensity. The data are expressed as the mean $\pm \mathrm{SD}$. ${ }^{*} \mathrm{P}<0.05$ vs. day 0 , $\mathrm{n}=5$. (B) Spearman's correlation analysis was used to determine the correlation between the expression levels of TRIP6 protein and miR-138-5p. Spearman's correlation, $\mathrm{r}=-0.7589(\mathrm{n}=20)$. (C) Immunofluorescence assay detected the expression of TRIP6 in NSCs (scale bar, $50 \mu \mathrm{m})$ and TRIP6 fluorescence intensity was quantified. "P<0.05 vs. miR-con group, ${ }^{*} \mathrm{P}<0.05$ vs. anti-miR-con group. (D) Schematic of miR-138-5p interaction with WT and Mut TRIP6 3'UTR. (E) Luciferase reporter assays in NSCs co-transfected with WT/Mut TRIP6 3'UTR reporter plasmid, along with miR-138-5p/anti-miR-138-5p or control mimic/inhibitor. The data are expressed as the mean \pm SD. " $\mathrm{P}<0.05$ vs. Mut TRIP6 3'UTR, n=5. NSCs, neural stem cells; SD, standard deviation; TRIP6, thyroid hormone receptor interacting protein 6; miR, microRNA; miR-con, control miR; WT, wild type; Mut, mutant. 
A

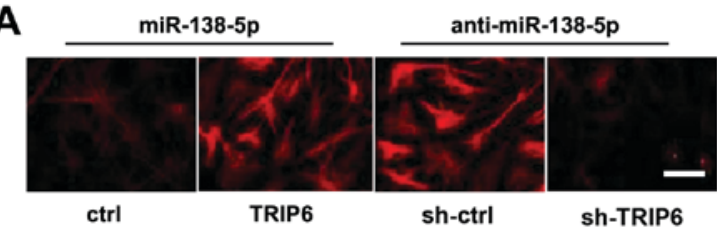

B

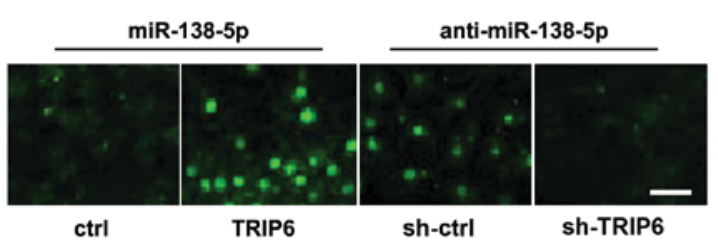

C

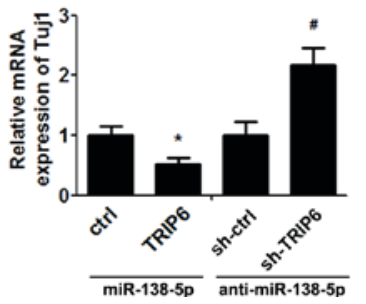

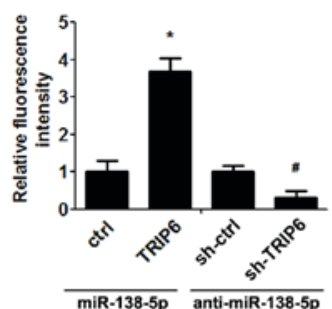
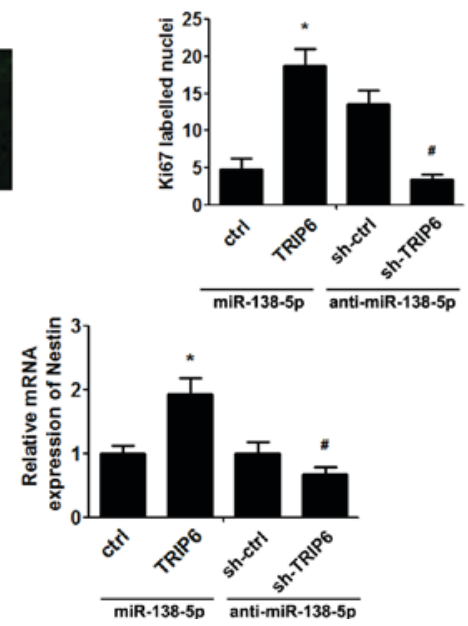

Figure 4. miR-138-5p exerts its biological effect via TRIP6. NSCs were co-transfected with miR-138-5p+ctrl vector, miR-138-5p+TRIP6 plasmid, anti-miR-138-5p+sh-ctrl vector or anti-miR-138-5p+sh-TRIP6 plasmid for $48 \mathrm{~h}$. (A) Immunofluorescence assay detected the expression of TRIP6 in NSCs (scale bar, $50 \mu \mathrm{m}$ ) and quantification of TRIP6 fluorescence intensity. The ctrl and sh-ctrl groups were both set as 1. (B) Proliferation of NSCs was detected by the Ki67 immunofluorescence (scale bar, $50 \mu \mathrm{m}$ ) and quantification of the number of Ki67-labelled nuclei by counting five high-power fields. (C) Quantitative polymerase chain reaction analysis of Tuj1 and nestin expression. The ctrl and sh-ctrl groups were both set as 1 . The data are expressed as the mean \pm standard deviation. ${ }^{*} \mathrm{P}<0.05$ vs. ctrl, ${ }^{\#} \mathrm{P}<0.05$ vs. sh-ctrl, $\mathrm{n}=5$. NSCs, neural stem cells; miR, microRNA; TRIP6, thyroid hormone receptor interacting protein 6 ; sh, short hairpin RNA; Tuj1, Tuj1, class III $\beta$-tubulin.

overexpression of miR-138-5p. By contrast, the activity of the luciferase reporter gene linked to the mutant TRIP6 3'UTR altered by the miR-138-5p mimic or anti-miR-138-5p (Fig. 3E).

miR-138-5p exerts its biological effect via TRIP6 expression. TRIP6 expression was upregulated and downregulated using a TRIP6 overexpression vector and sh-TRIP6, respectively, to determine whether TRIP6 is a functional target of miR-138-5p in NSCs. As presented in Fig. 4A, in NSCs transfected with miR-138-5p TRIP6 protein expression was increased by co-transfection with TRIP6-overexpressing plasmid, whereas reduced TRIP6 protein expression was observed in NSCs transfected with anti-miR-138-5p co-transfected shTRIP6 (Fig. 4A). Functionally, restoration of TRIP6 expression overturned the effect of miR-138-5p mimic, resulting in an increase in cell proliferation (Fig. 4B) and decrease of cell differentiation (Fig. 4C). By contrast, transfection with shTRIP6 reversed the effect of anti-miR-138-5p on NSCs proliferation (Fig. 4B) and differentiation (Fig. 4C).

\section{Discussion}

In recent years, research has focused on the profound importance of miRNAs in regulating neurogenesis. Increasing evidence supports that miRNAs are associated with the regulation of NSC proliferation and differentiation, and manipulating miRNAs in NSCs may be useful for the development of novel interventions for the treatment of certain neurological disorders $(15,16)$. The precise network of miRNAs that regulate neuronal proliferation and differentiation remains unclear.

Up or downregulation of miR-138 is important for regulation of the growth and/or apoptosis of various cancer types, including lung cancer (17), hepatocellular carcinoma (18) and leukaemia (19). miR-138-5p, the most common human isoform of miR-138, was previously demonstrated to be significantly downregulated in primary human pancreatic cancer and human pancreatic cancer-derived cell lines $(20,21)$. Exogenous overexpression of miR-138-5p inhibits pancreatic cancer cell growth (21). miR-138 is particularly well investigated in neuroscience, and has been suggested to have a potential role in mammalian brain function $(22,23)$. It was also reported that miR-138-5p acts as a molecular regulator of human memory function and dendritic spines, and regulates phosphorylation of tau protein, which may improve cognition and Alzheimer disease (24). However, the further association of miR-138-5p and proliferation and differentiation of NSCs has not yet been identified. In the current study, it was demonstrated that there was a gradual decline in the expression of miR-138-5p during the NSC differentiation. Suppression of miR-138-5p induced proliferation and reduced differentiation of NSCs, and overexpression of miR-138-5p reduced NSCs proliferation and promoted NSCs differentiation. These results indicated an important role of miR-138-5p in NSCs proliferation and differentiation.

Subsequently, it was elucidated that miR-138-5p targets and regulates TRIP6. TRIP6 was originally identified as an 
interacting protein of the nuclear thyroid hormone receptor in a yeast two-hybrid system, and as a member of the zyxin family of LIM proteins $(25,26)$. TRIP6 is a focal adhesion molecule with the ability to shuttle between the cell surface and nucleus, which is involved in the regulation of actin dynamics and signal transduction during in cell adhesion and migration (27). Accumulating evidence supports that TRIP6 is expressed in hippocampal neurons and modulates neurological biological function $(14,28)$. A previous study demonstrated that TRIP6 is necessary and sufficient for the self-renewal and proliferation of NSCs, but inhibited their differentiation (13). The results of the current study are in line with this previous conclusion, indicating that TRIP6 regulates NSC maintenance and it may be a novel marker for NSCs. In addition, the effect of miR-138-5p on proliferation and differentiation of NSCs was demonstrated be reversed by up- or downregulation of TRIP6 in the present study, which suggested that miR-138-5p regulates NSCs proliferation and differentiation, at least in part through modulating TRIP6 expression. These data also imply that TRIP6 is crucial for regulation of the balance between proliferation and differentiation of NSCs. Indeed, regarding the treatment of neurodevelopmental disorders, TRIP6 may be as a promising target for modulating NSCs.

In conclusion, the current study regards miR-138-5p as an important miRNA involved in the regulation of NSC proliferation and differentiation via targeting TRIP6. Therefore, altering miR-138-5p and TRIP6 regulation may be a promising treatment for dealing with neurogenesis and neurodegenerative diseases.

\section{Acknowledgements}

This project was supported by the National Natural Science Foundation (grant no. 31401258) and the Natural Science Foundation of Shandong Province, China (grant no. ZR2012BM006).

\section{References}

1. Liu S, Yin F, Zhang J, Wicha MS, Chang AE, Fan W, Chen L, Fan M and Li Q: Regulatory roles of miRNA in the human neural stem cell transformation to glioma stem cells. J Cell Biochem 115: 1368-1380, 2014.

2. Chaker Z, Codega P and Doetsch F: A mosaic world: Puzzles revealed by adult neural stem cell heterogeneity. Wiley Interdiscip Rev Dev Biol 5: 640-658, 2016.

3. Chung SY, Kishinevsky S, Mazzulli JR, Graziotto J, Mrejeru A, Mosharov EV, Puspita L, Valiulahi P, Sulzer D, Milner TA, et al: Parkin and PINK1 patient iPSC-derived midbrain dopamine neurons exhibit mitochondrial dysfunction and $\alpha$-synuclein accumulation. Stem Cell Reports 7: 664-677, 2016.

4. Cheng A, Hou Y and Mattson MP: Mitochondria and neuroplasticity. ASN Neuro 2: e00045, 2010.

5. Rao P, Benito E and Fischer A: MicroRNAs as biomarkers for CNS disease. Front Mol Neurosci 6: 39, 2013.

6. Shi X, Yan C, Liu B, Yang C, Nie X, Wang X, Zheng J, Wang Y and Zhu Y: miR-381 regulates neural stem cell proliferation and differentiation via regulating Hes1 expression. PLoS One 10: e0138973, 2015.

7. Ryu JR, Hong CJ, Kim JY, Kim EK, Sun W and Yu SW: Control of adult neurogenesis by programmed cell death in the mammalian brain. Mol Brain 9: 43, 2016.
8. Shukla GC, Singh J and Barik S: MicroRNAs: Processing, maturation, target recognition and regulatory functions. Mol Cell Pharmacol 3: 83-92, 2011.

9. Bartel DP: MicroRNAs: Target recognition and regulatory functions. Cell 136: 215-233, 2009.

10. Meza-Sosa KF, Pedraza-Alva G and Pérez-Martínez L: microRNAs: Key triggers of neuronal cell fate. Front Cell Neurosci 8: 175, 2014.

11. Palmer TD, Markakis EA, Willhoite AR, Safar F and Gage FH: Fibroblast growth factor-2 activates a latent neurogenic program in neural stem cells from diverse regions of the adult CNS. J Neurosci 19: 8487-8497, 1999.

12. Livak KJ and Schmittgen TD: Analysis of relative gene expression data using real-time quantitative PCR and the 2(-Delta Delta C(T)) method. Methods 25: 402-408, 2001.

13. Lai YJ, Li MY, Yang CY, Huang KH, Tsai JC and Wang TW: TRIP6 regulates neural stem cell maintenance in the postnatal mammalian subventricular zone. Dev Dyn 243: 1130-1142, 2014.

14. Lv K, Chen L, Li Y, Li Z, Zheng P, Liu Y, Chen J and Teng J: Trip6 promotes dendritic morphogenesis through dephosphorylated GRIP1-dependent myosin VI and F-actin organization. J Neurosci 35: 2559-2571, 2015.

15. Huang Y, Liu X and Wang Y: MicroRNA-378 regulates neural stem cell proliferation and differentiation in vitro by modulating Tailless expression. Biochem Biophys Res Commun 466: 214-220, 2015.

16. Tandon PN and Seth P: Cell therapy for neurological disorders: The elusive goal. Neurol India 64: 612-623, 2016.

17. Xiao L, Zhou H, Li XP, Chen J, Fang C, Mao CX, Cui JJ, Zhang W, Zhou HH, Yin JY and Liu ZQ: MicroRNA-138 acts as a tumor suppressor in non small cell lung cancer via targeting YAP1. Oncotarget 7: 40038-40046, 2016.

18. Liu Y, Zhang W, Liu K, Liu S, Ji B and Wang Y: miR-138 suppresses cell proliferation and invasion by inhibiting SOX9 in hepatocellular carcinoma. Am J Transl Res 8: 2159-2168, 2016.

19. Xu C, Fu H, Gao L, Wang L, Wang W, Li J, Li Y, Dou L, Gao X, Luo X, et al: BCR-ABL/GATA1/miR-138 mini circuitry contributes to the leukemogenesis of chronic myeloid leukemia. Oncogene 33: 44-54, 2014.

20. Tian S, Guo X, Yu C, Sun C and Jiang J: miR-138-5p suppresses autophagy in pancreatic cancer by targeting SIRT1. Oncotarget 8: 11071-11082, 2017.

21. Yu C, Wang M, Li Z, Xiao J, Peng F, Guo X, Deng Y, Jiang J and Sun C: MicroRNA-138-5p regulates pancreatic cancer cell growth through targeting FOXC1. Cell Oncol (Dordr) 38: 173-181, 2015.

22. Schroder J, Ansaloni S, Schilling M, Liu T, Radke J, Jaedicke M, Schjeide BM, Mashychev A, Tegeler C, Radbruch H, et al: MicroRNA-138 is a potential regulator of memory performance in humans. Front Hum Neurosci 8: 501, 2014.

23. Castaneda P, Muñoz M, Garcia-Rojo G, Ulloa JL, Bravo JA, Márquez R, García-Pérez MA, Arancibia D, Araneda K, Rojas PS, et al: Association of N-cadherin levels and downstream effectors of Rho GTPases with dendritic spine loss induced by chronic stress in rat hippocampal neurons. J Neurosci Res 93: 1476-1491, 2015.

24. Wang X, Tan L, Lu Y, Peng J, Zhu Y, Zhang Y and Sun Z: MicroRNA-138 promotes tau phosphorylation by targeting retinoic acid receptor alpha. FEBS Lett 589: 726-729, 2015.

25. Lin VT and Lin FT: TRIP6: An adaptor protein that regulates cell motility, antiapoptotic signaling and transcriptional activity. Cell Signal 23: 1691-1697, 2011.

26. Willier S, Butt E, Richter GH, Burdach S and Grunewald TG: Defining the role of TRIP6 in cell physiology and cancer. Biol Cell 103: 573-591, 2011.

27. Grunewald TG, Willier S, Janik D, Unland R, Reiss C, Prazeres da Costa O, Buch T, Dirksen U, Richter GH, Neff F, et al: The Zyxin-related protein thyroid receptor interacting protein 6 (TRIP6) is overexpressed in Ewing's sarcoma and promotes migration, invasion and cell growth. Biol Cell 105: 535-547, 2013.

28. Hoffman LM, Nix DA, Benson B, Boot-Hanford R, Gustafsson E, Jamora C, Menzies AS, Goh KL, Jensen CC, Gertler FB, et al: Targeted disruption of the murine zyxin gene. Mol Cell Biol 23: 70-79, 2003. 